(99.4\%), negative predictive value (91.1\%), sensitivity $(90.3 \%)$ and specificity $(99.4 \%)$ of the criteria led the authors to conclude that, although they could be improved, the preliminary criteria and the international registry are valuable epidemiologic tools.

Rebecca Doherty

Original article Cervera R et al. (2005) Validation of the preliminary criteria for the classification of catastrophic antiphospholipid syndrome. Ann Rheum Dis 64: 1205-1209

\section{A new treatment strategy for severe refractory rheumatoid arthritis}

High-dose chemotherapy (HDC) followed by autologous hematopoietic stem-cell transplantation (HSCT) is a new treatment strategy for patients with severe rheumatoid arthritis (RA) that is refractory to drug treatment. Although short-term studies had demonstrated significant improvement in signs and symptoms, the long-term impact of treatment had not been explored.

In this small Dutch study, Teng et al. followed eight patients (one male, seven female) who had undergone HDC and HSCT as part of a multicenter phase II trial. All had disease that was refractory to disease-modifying antirheumatic drugs and four had not responded to tumornecrosis-factor blockade. Patients were assessed every 3 months in the first year, every 6 months in the second year and once more at 5 years.

Good clinical improvement was noted in four patients 6 months after treatment; a further two improved moderately and two did not improve. By 2-year follow-up, all patients had relapsed and were using antirheumatic medications; however, response to these medications was restored. Functionality and health status showed a short-term improvement, but had declined by the 5-year point, although health status remained above that seen at baseline. Analysis of the quality-adjusted life-years gained using conventional RA therapy compared with HDC plus HSCT, confirmed that (when treatmentrelated mortality is less than $2.8 \%$ ) HDC plus HSCT is superior to conventional treatment.

Rebecca Doherty

Original article Teng YKO et al. (2005) Long-term followup of health status in patients with severe rheumatoid arthritis after high-dose chemotherapy followed by autologous hematopoietic stem cell transplantation. Arthritis Rheum 52: 2272-2276

\section{C-reactive protein predicts cardiovascular death in patients with inflammatory polyarthritis}

A prospective single-center study has found a correlation between levels of $\mathrm{C}$-reactive protein (CRP) measured at the time of diagnosis of inflammatory polyarthritis (IP) and death from cardiovascular disease (CVD). Goodson et al. had previously demonstrated increased CVD mortality rates in women with rheumatoidfactor (RF)-positive IP and aimed to extend this work by examining whether the level of CRP, a marker of inflammation, could independently predict CVD mortality rates in IP patients.

The group used data from death certificates to determine cause of death (median followup 10.1 years) for 506 patients recruited to an arthritis register between 1990 and 1992. Registered patients had definite inflammatory synovitis in two or more joints, lasting at least 4 weeks, and not subsequently explained by another, nonarthritic, diagnosis. Levels of CRP and RF were measured at baseline.

Multivariate analysis, adjusted for demographic and disease-related potential confounders, demonstrated that the presence of RF and a CRP level at or above $5 \mathrm{mg} / \mathrm{l}$ were strong and independent predictors of CVD mortality rates. Although comorbid CVD was defined by use of cardiovascular medication rather than assessed directly, the correlation between CRP level and CVD mortality rate was still present after adjusting for this factor.

Although the role of CRP in CVD is unclear, its identification as a predictive marker of CVD in patients with IP might allow better targeting of interventions for those at risk.

Rebecca Doherty

Original article Goodson NJ et al. (2005) Baseline levels of C-reactive protein and prediction of death from cardiovascular disease in patients with inflammatory polyarthritis. Arthritis Rheum 52: 2293-2299

\section{Harm is under-reported in rheumatic disease trials}

A recent systematic review has found that harm is inadequately reported in randomized, controlled trials assessing treatments for rheumatic disease. Reporting of harm was particularly poor in trials of nonpharmacologic treatment modalities. 